\title{
Accuracy Limits of Embedded Smart Device Accelerometer Sensors
}

\author{
Ahmed Ibrahim, Ahmed Eltawil, Yunsu Na and Sherif El-Tawil
}

\begin{abstract}
Smartphones are an indispensable tool in modern day-to-day life. Their widespread use has spawned numerous applications targeting diverse domains such as bio-medical, environment sensing and infrastructure monitoring. In such applications, the accuracy of the sensors at the core of the system is still questionable, since these devices are not originally designed for high accuracy sensing purposes. In this work, we investigate the accuracy limits of one of the commonly used sensors, namely, smartphone accelerometer. We focus on the efficacy of a smartphone as an acceleration measuring device, rather than focusing only on the accuracy of its internal accelerometer chip. This holistic approach includes additional errors that arise from the device operating system such as sampling time uncertainty. Hence, we propose a novel smart device accelerometer error model that includes the traditional additive noise as well as sampling time uncertainty errors represented by a white Gaussian process. The model is validated experimentally using shake table experiments, and maximum likelyhood estimation (MLE) is used to estimate the sampling time uncertainty standard deviation. Moreover, we derive the Cramer-Rao lower bound (CRLB) of acceleration estimation based on the proposed model.
\end{abstract}

Keywords - smartphone; accelerometer; error model; estimation; MLE.

\section{INTRODUCTION}

With the advent of the smart devices era, numerous applications (apps) have emerged that monitor a wide range of phenomena, ranging from human activities and vitals to environment sensing and infrastructure monitoring. The driving factor behind these innovative apps is the availability of sensors such as inertial (accelerometers and gyroscopes), pressure, temperature, humidity, light and proximity sensors.

One of the commonly used smartphone sensors is the inertial sensing module which contains tri-axial accelerometers and gyroscopes. Accelerometers in general are capable of measuring the force accompanying motion and according to Newton's second law of mechanics that force is proportional to acceleration. Several apps utilize smartphone tri-axial accelerometers in bio-medical applications to detect or monitor diseases. In [1], a smartphone-based app that is used to assess upper limb tremor in Parkinson's disease (PD) patients has been developed. That app assists physicians remotely to evaluate the patient's condition. Depending on a smartphone in such

This work was supported by National Science Foundation under Award Numbers CMMI:1362547, CMMI:1362458 and OAC:1638186.

A. Ibrahim is with the Electrical Engineering and Computer Science (EECS) department, University of California, Irvine, CA 92697-2625 (email: amibrah1@uci.edu)

A. Eltawil is with King Abdullah University of Science and Technology (KAUST), Thuwal, Saudia Arabia and the University of California, Irvine, CA 92697-2625 (email: aeltawil@ieee.org)

Y. Na and S. El-Tawil are with the Civil and Environmental Engineering department, University of Michigan, Ann Arbor, MI 48109-2125 (email: \{yunsu,eltawil\}@umich.edu) situations outperforms any other biomedical device in terms of cost, since no special hardware is needed. In [2], PD patients' actions have been identified by analyzing their speech and movement patterns. Smartphones in their pocket at different times of the day capture movement through the accelerometer chip. Several machine learning classification algorithms have been applied to classify patients as being Parkinson positive or negative. In [3], atrial fibrillation (AFib) detection has been proposed. A smartphone is placed on the chest of the patient and performs a noninvasive recording. No external sensors are needed yielding a very low cost approach. Using this method, it is possible to detect paroxysmal (or "silent") AFib. In [4], regular activities are detected and recorded as a main indicator of health. Data is captured by smartphones inertial sensors, then an online classifier differentiates between five common activities: cycling, running, sitting, standing, and walking. Monitoring these activities can effectively prevent and manage chronic diseases. Moreover, in [5], [6] an approach for the monitoring of elders and people with neurological pathologies (e.g., Alzheimer) has been proposed. Multi-sensor approaches are used to recognize critical events, such as falls or prolonged inactivity, that can be used to alert caregivers. Algorithms have been developed and applied on data provided by inertial sensors embedded in the user device. Other than alerts, the information provided by the proposed system can be used to track the evolution of diseases, and to track rehabilitation progress. In [7], smart watch accelerometers have been used to capture workout data which can assist users and physicians in monitoring health status and rehabilitation tasks. The proposed framework MiLift, automatically performs exercise identification, in addition to start/stop detection to remove user burdens of manual data entry.

On the other hand, smartphone apps also target environment and public safety monitoring. For instance, a crowdsensing framework for monitoring bridge vibrations using moving smartphones was presented in [8]. It was shown that acceleration data collected using smartphones in moving vehicles contain information about damage indicators such as the first three modal frequencies of the bridge under test. In addition, in [9], [10], [11], [12] structural health monitoring using a network of smartphones was proposed. A mobile app was developed that utilizes the phones internal accelerometers to sense earthquake vibration and assess building damage status. An early earthquake warning system (MyShake) was developed using only mobile sensors in [13]. MyShake successfully provided a 20 seconds warning for a magnitude 5 (M5) earthquake. Furthermore, community seismic network (CSN), another earthquake detection system used phone accelerometers to capture the s-wave in addition to the much harder to 
detect p-wave [14]. In [15], a crowd sensing application to estimate road conditions (CRATER) was presented. The app uses accelerometer data to detect speed bumps and potholes, and reports the locations to a cloud-based application.

In addition, smartphones have been used in intelligent transportation applications. For instance, in [16] movement detection is achieved based on a mobile device accelerometer found in a vehicle. Accelerometer is also used to detect motion events and trigger other sensors to capture data. Hence, a driving risk score is calculated based on the sensors data. Feedback notification is generated when the calculated risk score is above a predetermined threshold. Similarly, in [17] smart device accelerometer is used to estimate, acceleration, orientation, shock, vibration, velocity, speed, and position of vehicles. The accelerometer is part of telematics data acquisition device, which is configured to collect telematics data of a vehicle as an indication of vehicle safety. Then, a higher layer algorithm calculates insurance premium quote based on at least one of the received health telematics data.

Smart device sensors are designed for a consumer electronics market, not as a specialized sensing system, thus it is natural to expect that the specifications are lower than standalone sensors. In this work, we study the accuracy limits of a smartphone accelerometer, and the same approach is applicable to other smart devices such as wearables and tablets. Inertial sensors error is modeled in previous work [18], [19] as: 1) Scale factor error, 2) constant bias, 3) nonlinearity and 4) additive random noise. While the first three error types are deterministic and can be compensated by calibration, the random noise cannot be calibrated and can only be characterized as mentioned in [20], [21]. However, using the above traditional error model for a smart device accelerometer (or other sensors as well) does not capture the operating system (OS) timing uncertainty. In [22], [23], it was shown that Android has some limitations to be considered as a real time OS, and consequently when a mobile app polls the internal accelerometer chip every constant time period, the execution is expected to have some uncertainty that strongly depends on the coding style. Hence, the main contributions of this work can be summarized as follows:

1) Proposing a novel and experimentally validated error model for smart device accelerometer that includes sampling time uncertainty.

2) Proposing a maximum likelyhood estimation (MLE) methodology to estimate the model parameters.

3) Showing that linear estimation accuracy is close to the best achievable estimation accuracy determined by the Cramer-Rao lower bound (CRLB).

The rest of the paper is organized as follows. In section II, the proposed error model is illustrated. Section III describes the experimental procedure used to validate the proposed model and shows the results. The CRLB of the proposed model is derived in section IV. Finally, the conclusions are drawn in section V.

\section{Smart Device Accelerometer Proposed Noise MODEL}

In this section smart device accelerometer error is modeled. We propose an error model that takes into account sampling time uncertainty as well as additive noise. Since, the additive noise parameters can be well estimated using power spectral density or Allan variance techniques illustrated in [20], [21], in this work we focus on estimating the sampling time uncertainty standard deviation (STD). In other words, we assume additive noise parameters are known and given. MLE is adopted to estimate the sampling time uncertainty STD.

\section{A. Notation}

Throughout the paper, vectors are denoted by boldface lowercase letters, e.g. $\mathbf{x}$, and matrices by boldface uppercase letters, e.g. A. We use $(\cdot)^{T}$ for transpose of a vector or a matrix. Vector elements are denoted by lowercase normal font letters with subscript that indicates the element index, e.g. $x_{i}$ represents the $i$ th element in vector $\mathbf{x}$. In addition, the $i$ th column of the matrix $\mathbf{A}$ is given by $\mathbf{A}[:, i]$. We also use $\mathbb{E}[\cdot]$ as an expectation operator. The probability density function (PDF) of single variable $y_{i}$ and a vector $\mathbf{y}$ are denoted as $p\left(y_{i} ; \mathbf{x}\right)$ and $p(\mathbf{y} ; \mathbf{x})$ respectively, where $\mathbf{x}$ is a nonrandom parameter vector. We use $\odot$ to denote the Hadamard multiplication operator. Identity matrix of $K$ elements is denoted by $I_{K}$. Finally, $\operatorname{diag}(\mathbf{x})$ is a diagonal matrix whose diagonal entries correspond to the elements in the vector $\mathbf{x}$ :

$$
\operatorname{diag}(\mathbf{x})[i, j]= \begin{cases}x_{i} & i=j \\ 0 & i \neq j\end{cases}
$$

\section{B. Model}

Most prior work modeled the non-deterministic inertial sensor error as additive noise and characterised it using either power spectral density or Allan variance techniques as mentioned in [20], [21]. Sampling time uncertainty was neglected due to the fact that using a stable clock and proper sampling, results in negligible sampling time jitter error. While this is true in a general case, it is not true in smartphone systems, since reading acceleration data using a smart device is done through the OS. As mentioned in section I, smart device OS such as Android has limitations to act as a real time OS. Hence, it is expected to have sampling time uncertainty while polling the sensors. Therefore, we propose an error model for smart device accelerometer that includes additive noise as well as sampling time jitter error.

As mentioned in [24], [25], let the measured acceleration at any sampling instant $i$ be calculated by (1).

$$
a_{i}=\alpha x\left(i T+z_{i}\right)+w_{i}
$$

where $x(t), T, z_{i}$ and $w_{i}$ are the true acceleration, sampling period, sampling time jitter and additive noise at time sample $i$ respectively, and $\alpha$ is the scale factor that is ideally equal to one. Without loss of generality, we use normalized sampling frequency, i.e. let $T=1$. Error due to sampling time jitter was introduced in [24] by using the first order terms of Taylor series expansion of (1) and ignoring higher order terms. 
However, we found that this approach leads to erroneous values at low values of $|\dot{x}|$, as will be illustrated later in this paper.

Taylor expansion of (1) is given by (2), where $\frac{d^{n}}{d t^{n}}($.$) is$ the $n t h$ derivative operator. Both noise sources $z$ and $w$ are modeled as zero mean Gaussian noise with variance $\sigma_{z}^{2}$ and $\sigma_{w}^{2}$ respectively [24]. Modeling timing error in Android has been studied in several previous work such as [23], and it was shown that there are inaccurate timestamps in the Android environment, that negatively affected program correctness and reliability. In addition, quantitative analysis of jitter performance on Android mobile devices was shown. However, previous work in this field focused on assessment of uncertainty from a quantitative perspective rather than probabilistic modeling. On the other hand, sampling time uncertainty has been modeled as white Gaussian process in other domains such as analog to digital converters (ADCs) as illustrated in [24], [25]. Thus, for mathematical tractability we adopt white Gaussian model for sampling time uncertainty in smartphones.

$$
a_{i}=\alpha x_{i}+w_{i}+\left.\alpha \sum_{n=1}^{\infty} \frac{1}{n !} \frac{d^{n} x(t)}{d t^{n}}\right|_{t=i} z_{i}^{n}
$$

For simplicity, let $\frac{d x}{d t}$, and $\frac{d^{2} x}{d t^{2}}$ be denoted as $\dot{x}$ and $\ddot{x}$ respectively. Based on the negligible terms of (2) we investigate the following two models:

1) M1 First order: neglect $n>1$ terms in (2).

2) M2 Second order: neglect $n>2$ terms in (2).

M1: In this model, higher order terms $(n>1)$ of $(2)$ are neglected, hence measured acceleration is expressed by (3), and the vector form is given by (4), where $\mathbf{Z}=\operatorname{diag}(\mathbf{z})$. The joint PDF of $\mathbf{a}$ is given by (5), where $\sigma_{i}^{2}$ is the error variance at sample $i$ expressed by (6). The log likelyhood (LL) function is given by (7).

$$
\begin{aligned}
& a_{i}=\alpha x_{i}+w_{i}+\alpha \dot{x}_{i} z_{i} \\
& \mathbf{a}=\alpha \mathbf{x}+\mathbf{w}+\alpha \mathbf{Z} \dot{\mathbf{x}} \\
&\left.p\left(\mathbf{a} ; \mathbf{x}, \dot{\mathbf{x}}, \sigma_{w}, \sigma_{z}\right)\right|_{M 1}= \\
& \prod_{i=0}^{K-1} \frac{1}{\sqrt{2 \pi \sigma_{i}^{2}}} \exp \left(\frac{-\left(a_{i}-\alpha x_{i}\right)^{2}}{2 \sigma_{i}^{2}}\right) \\
& \sigma_{i}^{2}=\alpha^{2} \dot{x}_{i}^{2} \sigma_{z}^{2}+\sigma_{w}^{2}
\end{aligned}
$$

$$
\begin{array}{r}
\left.L L\left(\mathbf{a} ; \mathbf{x}, \dot{\mathbf{x}}, \sigma_{w}, \sigma_{z}\right)\right|_{M 1}=\log \left(p\left(\mathbf{a} ; \mathbf{x}, \dot{\mathbf{x}}, \sigma_{w}, \sigma_{z}\right)\right)= \\
-\frac{1}{2} \sum_{i=0}^{K-1}\left(\log \left(2 \pi \sigma_{i}^{2}\right)+\frac{\left(a_{i}-\alpha x_{i}\right)^{2}}{\sigma_{i}^{2}}\right)
\end{array}
$$

M2: In this model, higher order terms $(n>2)$ of (2) are neglected, hence measured acceleration is expressed by (8), and the vector form is given by (9). The joint PDF of $\mathbf{a}$ is given by (10). $p\left(a_{i} ; x_{i}, \dot{x}_{i}, \ddot{x}_{i}, \sigma_{w}, \sigma_{z}\right)$ is calculated by (11) where $*$ is the convolution operator, $\mathcal{N}\left(0, \sigma_{w}^{2}\right)$ is a zero mean normal distribution with variance $\sigma_{w}^{2}$ and $y_{i}$ is a random variable defined by (12). Since the PDF of $z$ is $\mathcal{N}\left(0, \sigma_{z}^{2}\right)$ and $y$ is a function of $z$ then $p\left(y_{i} ; \dot{x}_{i}, \ddot{x}_{i}, \sigma_{z}\right)$ is calculated numerically as described in [26]. The LL function of $\mathbf{M 2}$ is given by (13).

$$
\begin{aligned}
& a_{i}=\alpha x_{i}+w_{i}+\alpha \dot{x}_{i} z+\alpha \frac{\ddot{x}_{i}}{2} z_{i}^{2} \\
& \mathbf{a}=\alpha \mathbf{x}+\mathbf{w}+\alpha \mathbf{Z} \dot{\mathbf{x}}+\frac{1}{2} \alpha \mathbf{Z Z} \ddot{\mathbf{x}} \\
& p\left(\mathbf{a} ; \mathbf{x}, \dot{\mathbf{x}}, \ddot{\mathbf{x}}, \sigma_{w}, \sigma_{z}\right)= \\
& \prod_{i=0}^{K-1} p\left(a_{i} ; x_{i}, \dot{x}_{i}, \ddot{x}_{i}, \sigma_{w}, \sigma_{z}\right) \\
& p\left(a_{i} ; x_{i}, \dot{x}_{i}, \ddot{x}_{i}, \sigma_{w}, \sigma_{z}\right)= \\
& \mathcal{N}\left(0, \sigma_{w}^{2}\right) * p\left(y_{i} ; \dot{x}_{i}, \ddot{x}_{i}, \sigma_{z}\right) \\
& y_{i}=\alpha \dot{x_{i}} z_{i}+\alpha \frac{\ddot{x_{i}}}{2} z_{i}^{2} \\
& \left.L L\left(\mathbf{a} ; \mathbf{x}, \dot{\mathbf{x}}, \ddot{\mathbf{x}}, \sigma_{w}, \sigma_{z}\right)\right|_{M 2}= \\
& \sum_{i=0}^{K-1} \log \left(p\left(a_{i} ; x_{i}, \dot{x}_{i}, \ddot{x}_{i}, \sigma_{w}, \sigma_{z}\right)\right)
\end{aligned}
$$

As mentioned in [24], MLE is applied to estimate the model parameters, i.e. $\sigma_{w}$ and $\sigma_{z}$. However, since $\sigma_{w}$ can be estimated while the device is at rest as described in [20], [21], hence, we assume $\sigma_{w}$ is known.

For M1 and M2 models, $\sigma_{z}$ is estimated using (14). Without assuming negligible additive noise, i.e. $\sigma_{w} \neq 0$, equation (14) has no closed form solution, hence we used a numerical approach as explained in section II-C.

$$
\hat{\sigma}_{z}=\underset{\sigma_{z}}{\operatorname{argmax}} L L\left(\mathbf{a} ; \mathbf{x}, \dot{\mathbf{x}}, \ddot{\mathbf{x}}, \sigma_{w}, \sigma_{z}\right)
$$

\section{Simulation Results}

In this section, we evaluate the efficacy of the models proposed in section II-B. A sinusoidal signal is used as the ground truth acceleration $x(t)$ whose normalized frequency is 0.04 and amplitude $10^{4} \mathrm{~mm} / \mathrm{s}^{2}$. Let the sampling clock have jitter of Gaussian distribution with zero mean and $\sigma_{z}=0.2$. For smartphone accelerometers, according to [20], [27], the additive noise typically has $\sigma_{w}=10 \mathrm{~mm} / \mathrm{s}^{2}$.

The simulation is repeated 1000 times to calculate the estimation accuracy of the proposed models. At $\alpha=1$, the exact error, M1 and M2 errors are calculated by (1517) respectively. Figure 1 compares between the root mean squared (RMS) of the exact error, M1 and M2 RMS errors. It is clear that:

1) The RMS error is greater than the additive noise level represented by $\sigma_{w}$.

2) Error is maximum at the points of highest $|\dot{x}|$ values, i.e. the points that have the fastest transitions.

3) M1 and M2 match the exact error RMS at high values of $|\dot{x}|$, however at low values, M1 underestimates the error. 


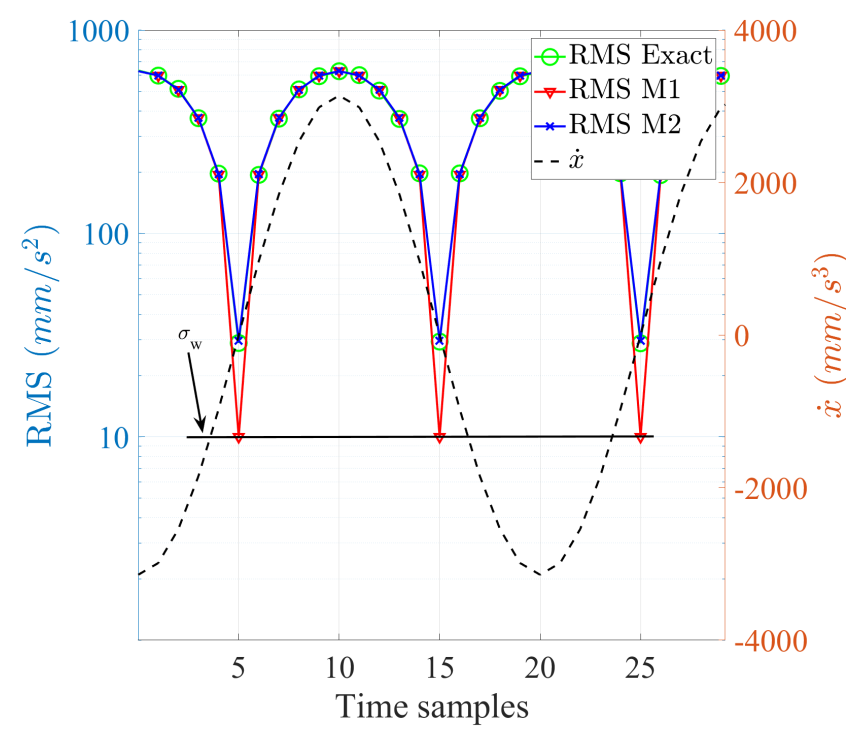

Fig. 1: Exact, M1 and M2 RMS (left axis). True first derivative of acceleration signal $\dot{x}$ (right axis).

$$
\begin{aligned}
e_{i} & =a_{i}-x_{i} \\
\left.e_{i}\right|_{M 1} & =w_{i}+\dot{x}_{i} z_{i} \\
\left.e_{i}\right|_{M 2} & =w_{i}+\dot{x}_{i} z_{i}+\frac{1}{2} \ddot{x}_{i} z_{i}^{2}
\end{aligned}
$$

In order to estimate $\sigma_{z}$ using MLE, equation (14) is solved numerically by sweeping on a range of values to get the $\sigma_{z}$ value that maximizes the LL function. That is repeated for a range of $\alpha$ values to consider the scale factor error that exists even at nominal temperature, and is reported by the sensor vendor. Figure 2 shows $\sigma_{z}$ estimation for M1 and $\mathbf{M} 2$ in case a slight scale factor error is introduced. It is clear that $\mathbf{M 2}$ is less sensitive to scale factor errors than M1. This is due to the fact that M2 is much more accurate at the low $|\dot{x}|$ values as mentioned earlier, and these points are the maximum values of $x$, i.e. the points that have the maximum error in case there is a scale factor error.

\section{EXPERIMENTS}

In order to validate the proposed models, shake table experiments were performed. In this section, the experiments are illustrated including the testing flow and the experimental setup. The main steps of the experiments are: 1) estimation of $\sigma_{z}$ using the proposed models M1 and M2,2) calculation of the RMS error based on these models and 3) comparing the calculated RMS to the actual measured error. Four different smartphones are used in the experiments: SMG900T, SMT350 and two different SMG920V devices. SMG900T and SMG920V are equipped with triaxial accelerometer chip manufactured by Invensense [27] and SMT350 includes STMicroelectronics accelerometer chip. The operating system running

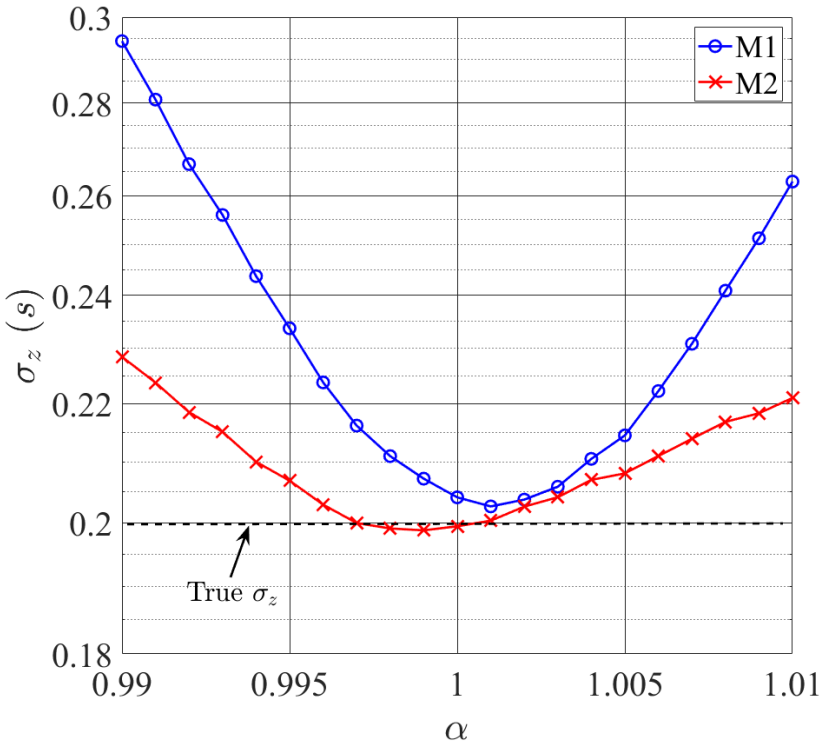

Fig. 2: M1 and M2 estimation bias due to scale factor error.

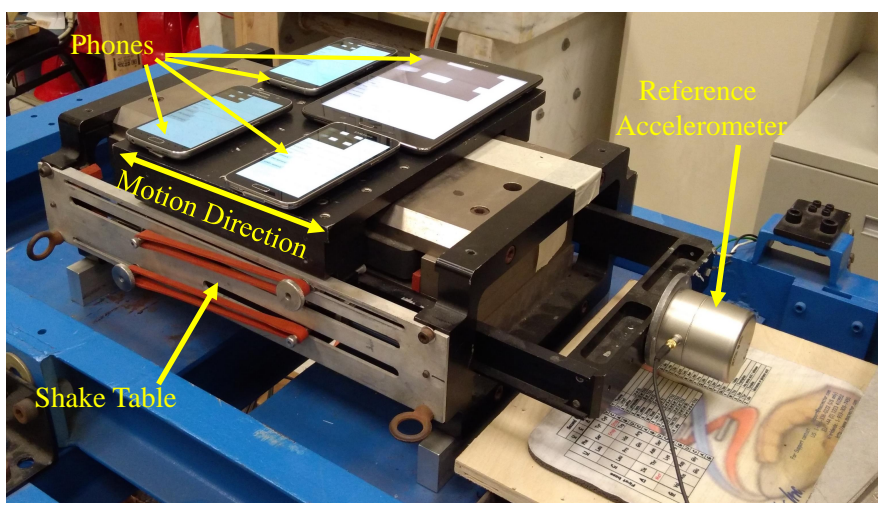

Fig. 3: Shake table experiment setup.

on the four phones is Android and the versions are summarized in table I. A seismic grade accelerometer PCB Piezotroincs Model393C [28] is used as a reference to measure the ground truth acceleration.

Five experiments are performed, where in each one the table is excited for 25 seconds by a sinusoidal signal of one of the following frequencies: 4, 5, 6, 7 and $8 \mathrm{~Hz}$. The four smart devices are mounted to the shake table to avoid sliding. The

TABLE I: Characteristics of smart devices used in the experiments.

\begin{tabular}{|c|c|c|c|}
\hline OS & SMG900T & SMG920V & SMT350 \\
\hline $\begin{array}{c}\text { Android } \\
\text { V5.1.1 }\end{array}$ & Android V7 & Android V6 \\
\hline $\begin{array}{c}\text { Accelerome- } \\
\text { ter Chip } \\
\text { manufacturer }\end{array}$ & Invensense & Invensense & $\begin{array}{c}\text { STMicroelec- } \\
\text { tronics }\end{array}$ \\
\hline $\begin{array}{c}\text { Commercial } \\
\text { Name }\end{array}$ & $\begin{array}{c}\text { Samsung } \\
\text { Galaxy S5 }\end{array}$ & $\begin{array}{c}\text { Samsung } \\
\text { Galaxy S6 }\end{array}$ & $\begin{array}{c}\text { Samsung } \\
\text { Galaxy Tab } \\
\text { A }\end{array}$ \\
\hline
\end{tabular}




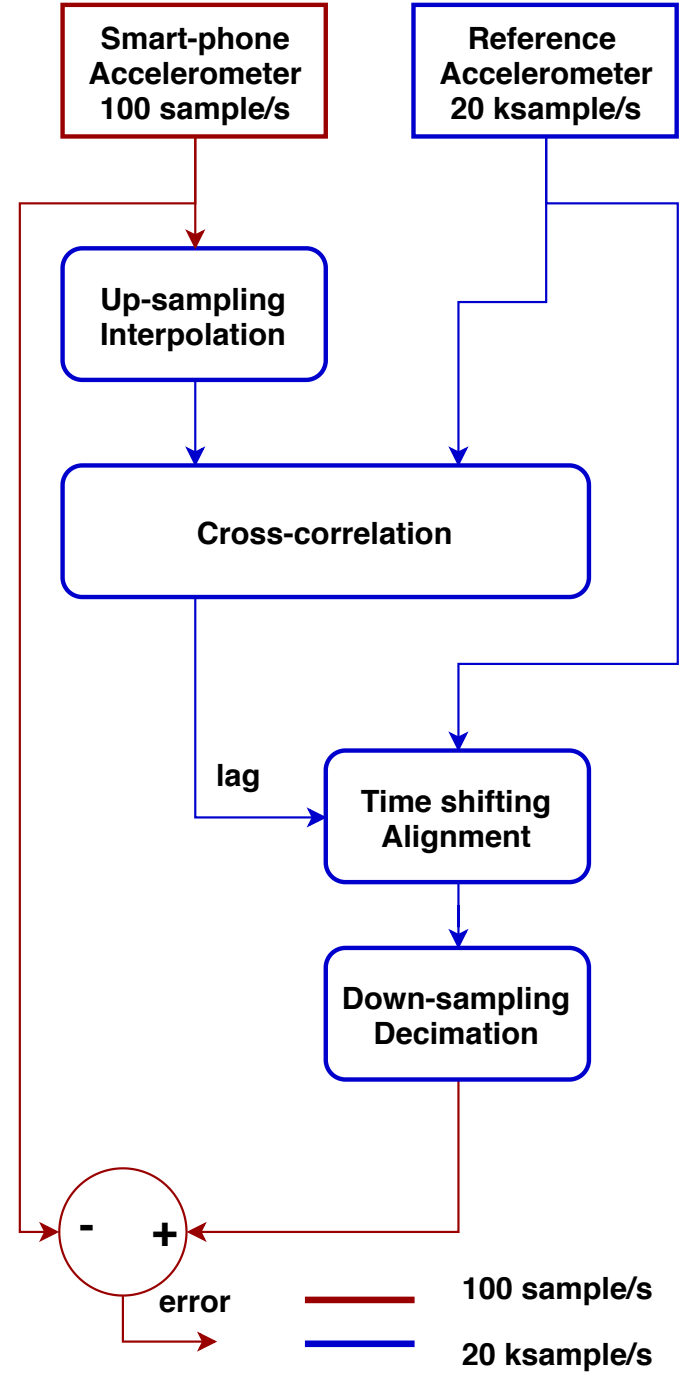

Fig. 4: Smartphone accelerometer error calculation flow chart. Phone acceleration is up-sampled to match the reference sampling rate. Cross correlation is used to align the phone data with the reference one. Reference is down-sampled to the phone nominal rate after alignment. Error is calculated by subtraction.

smart devices are sampling at 100 samples/s rate, i.e. for each 25 -sec experiment 2500 acceleration samples are stored. Figure 3 shows the experiment setup. We selected this specific range of frequencies for the experiments based on the region of interest of the target applications. For instance frequency of tremors of a Parkinson's disease (or similar diseases) patients are between $5-8 \mathrm{~Hz}$ [29]. Human activities frequency spectrum is between 0 and $20 \mathrm{~Hz}$, with about $98 \%$ of the Fourier transform amplitude is below $10 \mathrm{~Hz}$ [30], and $95 \%$ of spectrum amplitude of gait is below $10 \mathrm{~Hz}$ [31]. For structural health monitoring, several previous work reported buildings natural frequency to be below $10 \mathrm{~Hz}$ [32], [33], [34].

Figure 4 shows the procedure followed to calculate the error of each smartphone. A nominal sampling rate of 100 samples/s is selected for the smartphones accelerometers, whereas the reference is sampled at $20 \mathrm{ksample} / \mathrm{s}$. First,
TABLE II: Summary of experimental results. Five experiments are performed to estimate the proposed model M1 parameter $\sigma_{z}$ for four different phones. Each experiment is a sinusoidal wave with certain frequency. The average $\sigma_{z}$ value is the square root of the average variance $\sigma_{z}^{2}$ of all the experiments.

\begin{tabular}{|c|c|c|c|c|}
\hline & \multicolumn{4}{|c|}{$\sigma_{z 1}(s)$} \\
\hline & SMG900T & SMG920V & $\begin{array}{c}\text { SMG920V } \\
\text { (2nd) }\end{array}$ & SMT350 \\
\hline Sine $4 \mathrm{~Hz}$ & 0.213 & 0.21 & 0.141 & 0.243 \\
\hline Sine $5 \mathrm{~Hz}$ & 0.3 & 0.25 & 0.1160 & 0.3 \\
\hline Sine $6 \mathrm{~Hz}$ & 0.239 & 0.187 & 0.12 & 0.272 \\
\hline Sine $7 \mathrm{~Hz}$ & 0.156 & 0.216 & 0.131 & 0.235 \\
\hline Sine $8 \mathrm{~Hz}$ & 0.214 & 0.208 & 0.131 & 0.235 \\
\hline \hline Average & 0.2292 & 0.2166 & 0.1248 & 0.2714 \\
\hline
\end{tabular}

TABLE III: Summary of experimental results. Five experiments are performed to estimate the proposed model M2 parameter $\sigma_{z}$ for four different phones. Each experiment is a sinusoidal wave with certain frequency. The average $\sigma_{z}$ value is the square root of the average variance $\sigma_{z}^{2}$ of all the experiments.

\begin{tabular}{|c|c|c|c|c|}
\hline & \multicolumn{4}{|c|}{$\sigma_{z 2}(s)$} \\
\hline & SMG900T & SMG920V & $\begin{array}{c}\text { SMG920V } \\
\text { (2nd) }\end{array}$ & SMT350 \\
\hline Sine $4 \mathrm{~Hz}$ & 0.187 & 0.185 & 0.131 & 0.208 \\
\hline Sine $5 \mathrm{~Hz}$ & 0.178 & 0.174 & 0.108 & 0.208 \\
\hline Sine $6 \mathrm{~Hz}$ & 0.179 & 0.171 & 0.116 & 0.212 \\
\hline Sine $7 \mathrm{~Hz}$ & 0.132 & 0.18 & 0.126 & 0.201 \\
\hline Sine $8 \mathrm{~Hz}$ & 0.163 & 0.167 & 0.109 & 0.2 \\
\hline \hline Average & 0.1689 & 0.1755 & 0.1184 & 0.2059 \\
\hline
\end{tabular}

the smartphone data is up-sampled by cubic interpolation to match the reference rate. Second, cross-correlation is used to estimate the time lag that maximizes the cross-correlation. The reference is shifted by the estimated lag and then downsampled to the nominal phone sampling rate. Finally, the error is calculated by subtraction. The procedure is repeated for each of the four phones in the five experiments.

We used the proposed MLE M1 and M2 to estimate $\sigma_{z}$ for each phone, and the results are summarized in tables II and III respectively. We denote M1 and M2 estimation results as $\sigma_{z 1}$ and $\sigma_{z 2}$ respectively. Based on the results, it is clear that that the estimated $\sigma_{z}$ values are not much varying across the experiments for a given device, which validates the assumption that this parameter is not signal dependent.

It is worth noting that the models described in section II have two use cases:

1) Characterization: where a known vibration signal is used to estimate $\sigma_{z}$.

2) Error estimation: where the model parameters $\sigma_{z}$ and $\sigma_{w}$ are known, i.e. already estimated in the characterization phase, and then the error level of measuring an unknown signal is to be estimated using the models.

Hence, for all the experiments, we compare the measurements 


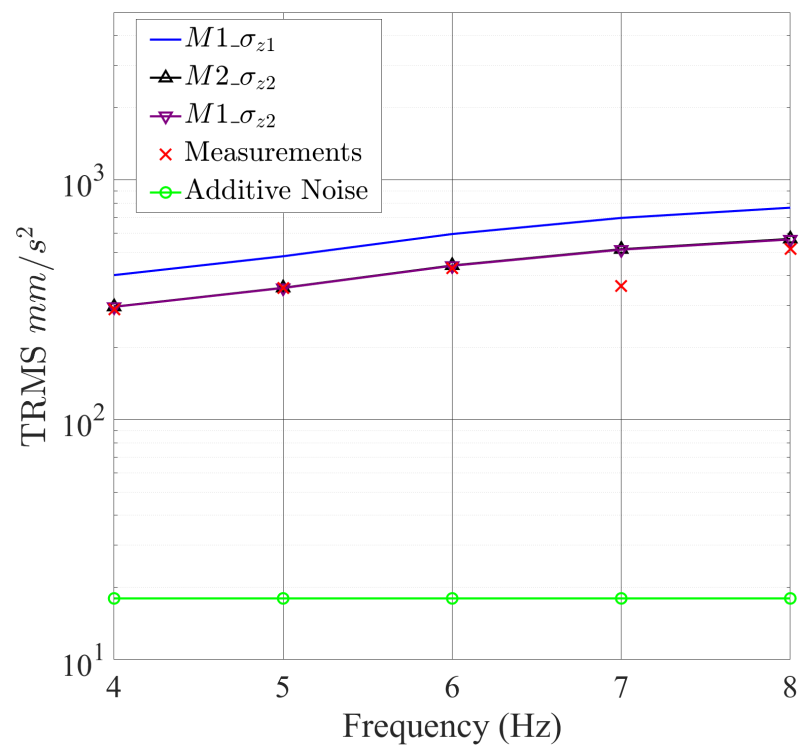

Fig. 5: TRMS error versus the frequency of shaking for $M 1 \_\sigma_{z 1}, M 2 \_\sigma_{z 2}$ and $M 1 \_\sigma_{z 2}$ compared to measurements of SMG900T.

to the following three techniques:

1) $M 1_{-} \sigma_{z 1}: \sigma_{z}$ is characterized by $\mathbf{M 1}$ and error is estimated by $\mathbf{M 1}$

2) $M 2_{-} \sigma_{z 2}: \sigma_{z}$ is characterized by $\mathbf{M 2}$ and error is estimated by $\mathbf{M} 2$

3) $M 1 \_\sigma_{z 2}: \sigma_{z}$ is characterized by $\mathbf{M 2}$ and error is estimated by $\mathbf{M 1}$

For the four phones, figures 5-8 compare the measured error represented by the time average root mean squared (TRMS) to $M 1 \_\sigma_{z 1}, M 2 \_\sigma_{z 2}$ and $M 1 \_\sigma_{z 2}$ models. We also added the traditional additive noise model to the figures which does not take into account sampling time jitter. It is clear that the closest models to the measurements are $M 2 \_\sigma_{z 2} M 1 \_\sigma_{z 2}$, which indicates the effectiveness of using $\mathbf{M} \mathbf{2}$ in the characterization phase. However, if $\sigma_{z}$ is properly estimated or given, then estimating the error using M1 and M2 result in almost the same values. Since, M1 is a less complex model, we believe $M 1 \_\sigma_{z 2}$ is the best technique.

As shown in the results, the proposed model and parameter estimation procedure is validated on devices running different operating system versions and different accelerometer chips. Since the proposed model has not assumed any special features of Android or the inertial module chip, we believe the same methodology of parameter estimation applies to other devices as well, however the jitter values might be different. For instance, devices running other operating systems such as iOS or Microsoft Windows might have another jitter performance. Nevertheless, characterizing that jitter can still be achieved using the methodology proposed in this work.

\section{CRLB FOR ACCELERATION ESTIMATION}

In this section we derive the CRLB of acceleration estimation based on the proposed model. The CRLB evaluates

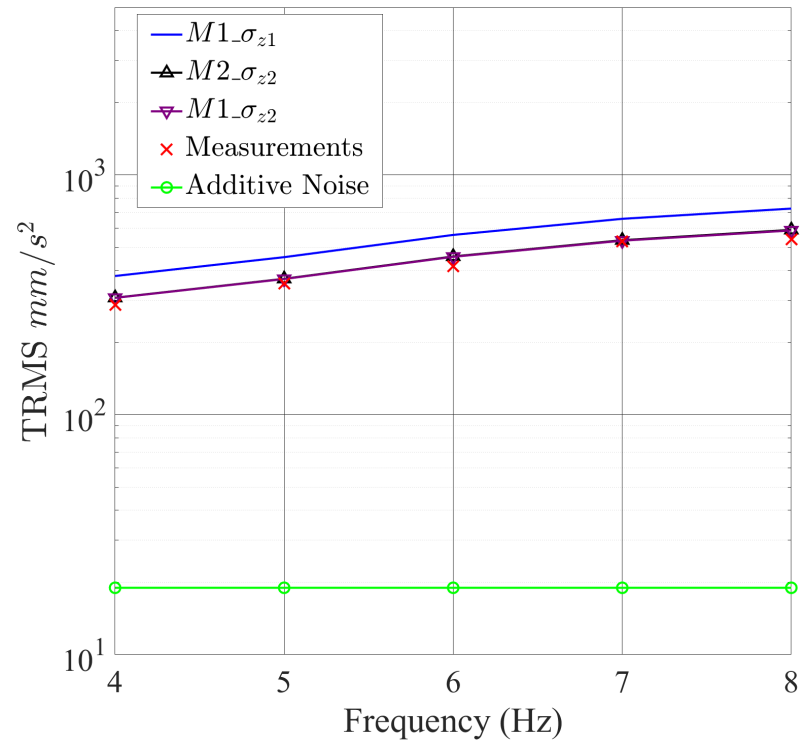

Fig. 6: TRMS error versus the frequency of shaking for $M 1 \_\sigma_{z 1}, M 2 \_\sigma_{z 2}$ and $M 1 \_\sigma_{z 2}$ compared to measurements of SMG920V.

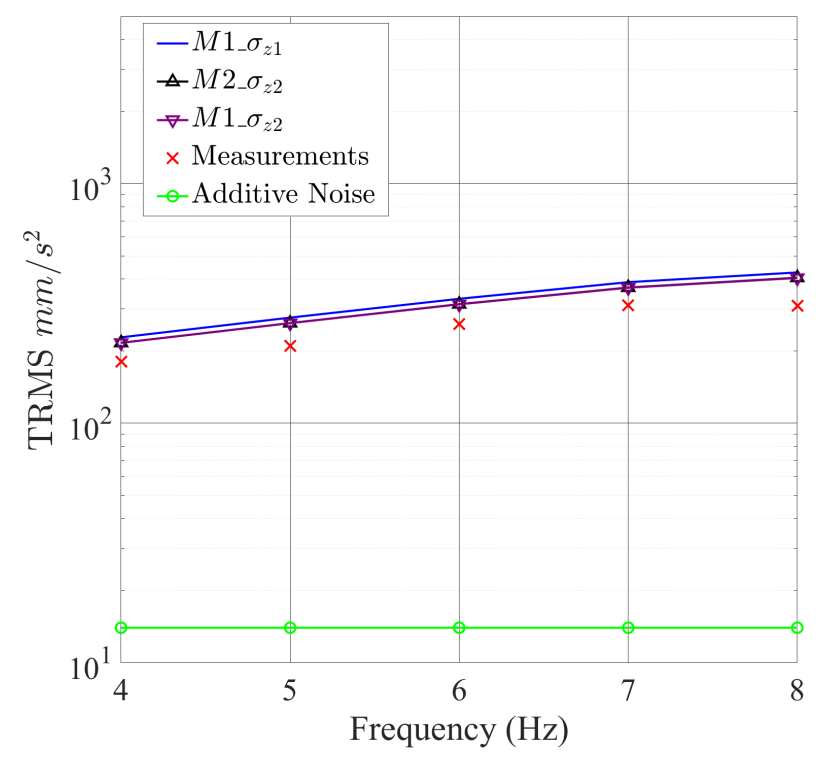

Fig. 7: TRMS error versus the frequency of shaking for $M 1 \sigma_{z 1}, M 2_{-} \sigma_{z 2}$ and $M 1_{-} \sigma_{z 2}$ compared to measurements of the second SMG920V. 


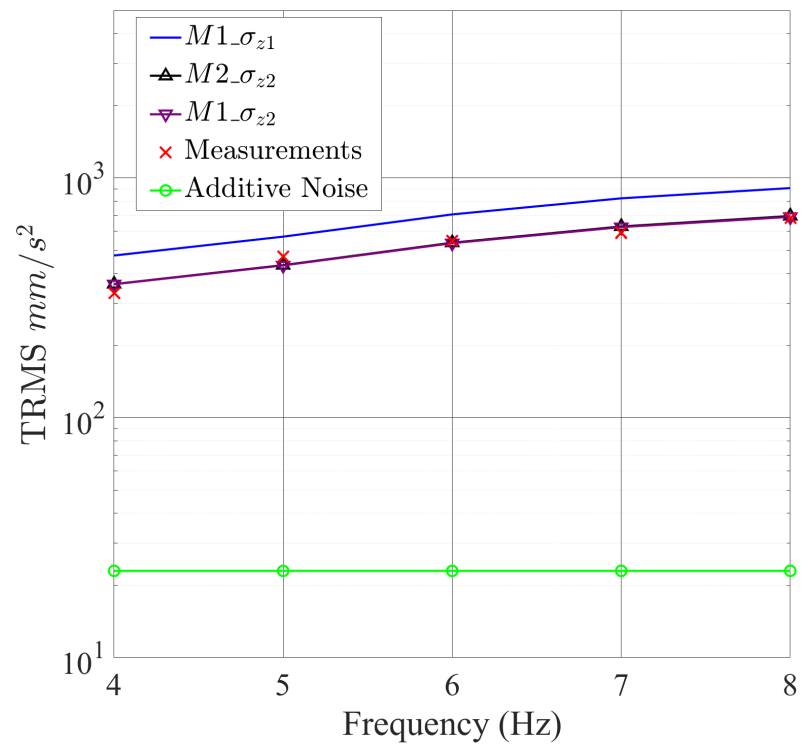

Fig. 8: TRMS error versus the frequency of shaking for $M 1 \_\sigma_{z 1}, M 2 \_\sigma_{z 2}$ and $M 1 \_\sigma_{z 2}$ compared to measurements of SMT350.

the best achievable estimation accuracy of unbiased estimators [35]. We assume that since M1 and M2 are almost equivalent in the TRMS, then we evaluate the CRLB of M1. In addition, since we are concerned about the best achievable accuracy, let $\alpha$ be equal to one.

As mentioned in [35], for any parameter vector $\boldsymbol{\theta}$ the CRLB for each element $\theta_{i}$ is defined by (18), where $\mathbf{I}(\boldsymbol{\theta})$ is the Fisher information matrix defined by (19).

$$
\begin{aligned}
\operatorname{var}\left(\theta_{i}\right) & \geq \mathbf{I}(\boldsymbol{\theta})^{-1}[i, i] \\
\mathbf{I}(\boldsymbol{\theta})[i, j] & =-\mathbb{E}\left[\frac{\partial^{2} \ln p(\mathbf{x} ; \boldsymbol{\theta})}{\partial \theta_{i} \partial \theta_{j}}\right]
\end{aligned}
$$

As mentioned in [25], nth derivative is approximately evaluated by (20), where $\operatorname{sinc}^{(n)}$ is the $n t h$ derivative of the cardinal sine function defined by (21), and $K$ is the number of time samples, refer to appendix A for more information. Consequently, (2) can be written as (22) and the vector form is expressed by (23), where $\mathbf{D}$ is a matrix whose rows are defined by (24).

$$
\begin{aligned}
\left.\frac{d^{n} x(t)}{d t^{n}}\right|_{t=i} & \approx \sum_{k=0}^{K-1} x_{k} \operatorname{sinc} c^{(n)}(i-k) \\
\operatorname{sinc}(t) & =\frac{\sin \pi t}{\pi t} \\
a_{i} & =x_{i}+w_{i}+\sum_{k=0}^{K-1} x_{k} \operatorname{sinc}(1)(i-k) z_{i} \\
\mathbf{a} & =\mathbf{x}+\mathbf{w}+\mathbf{Z D \mathbf { x }}
\end{aligned}
$$

Hence, as described in [25], each element of the Fisher information matrix (FIM) is evaluated by (25), where the covariance matrix of $\mathbf{a}$ is given by (26) and its derivative with

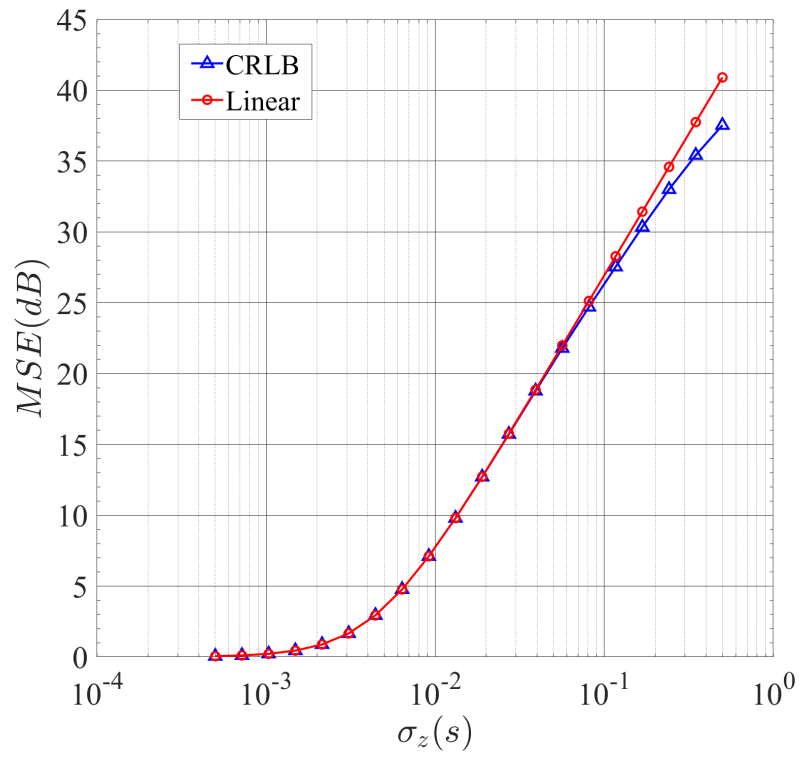

Fig. 9: MSE of linear estimation of acceleration is plotted versus $\sigma_{z}$ and compared to the CRLB, knowing that the true acceleration signal is a sinusoidal signal of $10^{4} \mathrm{~mm} / \mathrm{s}^{2}$ amplitude and $0.04 \mathrm{~Hz}$ normalized frequency. The zero-dB level corresponds to the additive noise.

respect to $x_{i}$ is given by (27). Then, according to the CRLB theorem, the minimum mean squared error (MSE) obtained by an unbiased estimator is given by (28).

$$
\begin{aligned}
& \mathbf{D}[i,:]=\left[\begin{array}{c}
\operatorname{sinc}^{(1)}(-i) \\
\operatorname{sinc}(1)(1-i) \\
\operatorname{sinc}(1)(2-i) \\
\vdots \\
\operatorname{sinc}(1)((K-1)-i)
\end{array}\right]^{T} \\
& \boldsymbol{F I M}[i, j]=\frac{1}{2} \operatorname{tr}\left\{\frac{\partial \boldsymbol{\Sigma}_{\boldsymbol{a}}}{\partial x_{i}} \boldsymbol{\Sigma}_{\boldsymbol{a}}{ }^{-1} \frac{\partial \boldsymbol{\Sigma}_{\boldsymbol{a}}}{\partial x_{j}} \boldsymbol{\Sigma}_{\boldsymbol{a}}^{-1}\right\}+\boldsymbol{\Sigma}_{\boldsymbol{a}}^{-1}[i, j] \\
& \boldsymbol{\Sigma}_{\boldsymbol{a}}=\sigma_{z}^{2} I_{K} \odot\left(\mathbf{D} \mathbf{x} \mathbf{x}^{T} \mathbf{D}^{T}\right)+\sigma_{w}^{2} I_{K} \\
& \frac{\partial \boldsymbol{\Sigma}_{\boldsymbol{a}}}{\partial x_{i}}=2 \sigma_{z}^{2} \operatorname{diag}\{\mathbf{D}[:, i] \odot(\mathbf{D x})\} \\
& \left.M S E\right|_{\min }=\mathbb{E}\left[(\hat{\mathbf{x}}-\mathbf{x})^{T}(\hat{\mathbf{x}}-\mathbf{x})\right]=\operatorname{tr}\left\{\mathbf{F I M}^{-1}\right\}
\end{aligned}
$$

The CRLB is evaluated numerically assuming ground truth acceleration is a sinusoidal signal of amplitude $10^{4} \mathrm{~mm} / \mathrm{s}^{2}$ and normalized frequency of $0.04 \mathrm{~Hz}, \sigma_{w}=10 \mathrm{~mm} / \mathrm{s}^{2}$ and different $\sigma_{z}$ values. Figure 9 compares the CRLB to the linear estimation accuracy. It is worth noting that a linear estimator as mentioned in [25], corresponds to $\hat{\mathbf{x}}=\mathbf{a}$, i.e. $\left.M S E\right|_{\text {Linear }}=$ $\operatorname{tr}\left\{\boldsymbol{\Sigma}_{\boldsymbol{a}}\right\}$ in case no over sampling is assumed.

As mentioned in section III, $\sigma_{z}$ is approximately about 0.2 , hence, it is clear from figure 9 that linear estimator accuracy is very close to the minimum theoretical bound. However, for higher jitter, other estimation techniques such as maximum likelihood estimation [25] can be adopted. 


\section{CONCLUSION}

Smart devices are equipped with multiple sensors that measure most of the surrounding physical quantities. Lots of apps emerged that target biomedical, environment and infrastructure applications. In this work, we have investigated the accuracy limits of a smart device accelerometer which is considered one of the most used sensors in such applications. We proposed a novel error model that takes into account software sampling time error in addition to the additive noise model developed in previous work. The proposed model has been validated experimentally on four smart devices by several shake table experiments. It was shown that the proposed model estimates error TRMS that is much closer to the real measured error compared to the additive noise model which is about an order of magnitude less. In addition, with respect to acceleration estimation accuracy based on the proposed model, it was shown that the linear estimators are close to the CRLB at the current sampling time jitter value. Hence, using a less computationally expensive linear estimator will not affect much the achieved accuracy.

\section{APPENDIX A}

\section{SignAl Derivative APPROXIMATION}

Equation (20) is used to get an approximate value of the signal derivative as a function of the signal itself. Based on Nyquist sampling theorem, any band limited signal $x(t)$ of bandwidth $B$ can be recovered from its sampled version $x_{i}$ if the sampling frequency $f_{s}$ is at least $2 B$. The signal reconstruction is done using an ideal low pass filter of bandwidth $B$. Without the loss of generality, we use normalized frequency, i.e. $f_{s}=1$. The low pass filter impulse response is defined by (29). Hence, $x(t)$ is recovered by (30). Then, the first derivative of $x(t)$ is calculated by (31), where $\operatorname{sinc}(1)(t)$ is the first derivative of $\operatorname{sinc}(t)$. It is worth noting that since $x(t)$ is band limited then it is infinitely long in the time domain. In addition, $\operatorname{sinc}(t)$ is also infinitely long. However, for practical implementation, both the signals are truncated which results in an approximation error. Let the approximated first derivative of $x(t)$ be denoted as $\tilde{\dot{x}}(t)$ which is calculated by (32) and the discrete version is expressed by (33).

$$
\begin{array}{r}
h(t)=\operatorname{sinc}(t) \\
x(t)=\sum_{k=-\infty}^{\infty} x_{k} \operatorname{sinc}(t-k) \\
\dot{x}(t)=\sum_{k=-\infty}^{\infty} x_{k} \operatorname{sinc} c^{(1)}(t-k) \\
\tilde{\dot{x}}(t)=\sum_{k=0}^{K-1} x_{k} \operatorname{sinc}^{(1)}(t-k) \\
\tilde{\dot{x}}_{i}=\sum_{k=0}^{K-1} x_{k} \operatorname{sinc}^{(1)}(i-k)
\end{array}
$$

Since the sinc unction is decreasing, the approximation error mainly affects the data points at the boundaries, in other words, the middle points are not much affected by the truncated part of the functions. For the sake of demonstration,

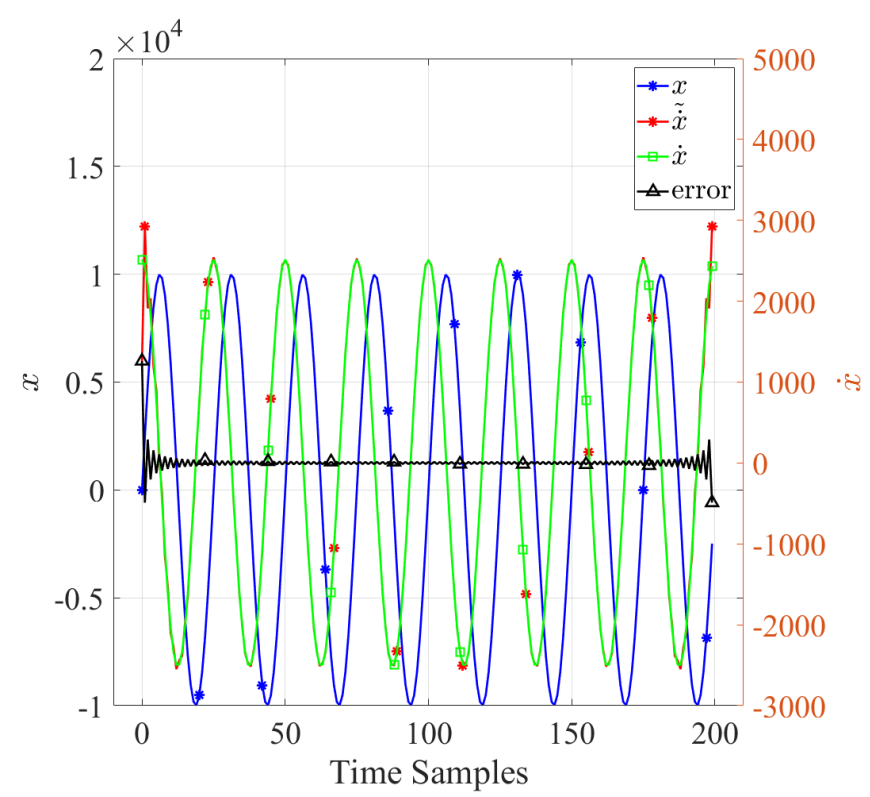

Fig. 10: True and approximate first derivative example.

let $K=200, \sigma_{z}=0.2$ and $x_{i}=10^{4} \sin (2 \pi 0.04 i)$ then the true and approximate derivatives are expressed by (34) and (35) respectively. Figure 10 compares between the true and approximate first derivatives of the given example and it is clear that the error is larger at the boundaries.

For the same example, the FIM is evaluated using (25), and the CRLB is the diagonal of FIM $^{-1}$. Figure 11 shows the CRLB at each sample time $i$. It is clear that at the boundaries the CRLB is different compared to the middle samples, which is expected due to the inaccurate signal derivative at the boundaries as mentioned earlier.

$$
\begin{aligned}
& \dot{x}_{i}=2 \pi 0.04 \times 10^{4} \cos (2 \pi 0.04 i) \\
& \tilde{\dot{x}}_{i}=\sum_{k=0}^{K-1} 10^{4} \sin (2 \pi 0.04 k) \operatorname{sinc} c^{(1)}(i-k)
\end{aligned}
$$

\section{REFERENCES}

[1] N. Kostikis, D. Hristu-Varsakelis, M. Arnaoutoglou, and C. Kotsavasiloglou, "A smartphone-based tool for assessing parkinsonian hand tremor," IEEE Journal of Biomedical and Health Informatics, vol. 19, no. 6, pp. 1835-1842, Nov 2015.

[2] S. Wan, Y. Liang, Y. Zhang, and M. Guizani, "Deep multi-layer perceptron classifier for behavior analysis to estimate parkinson's disease severity using smartphones," IEEE Access, vol. 6, pp. 36825-36833, 2018.

[3] O. Lahdenoja, T. Hurnanen, Z. Iftikhar, S. Nieminen, T. Knuutila, A. Saraste, T. Kiviniemi, T. Vasankari, J. Airaksinen, M. Pänkäälä, and T. Koivisto, "Atrial fibrillation detection via accelerometer and gyroscope of a smartphone," IEEE Journal of Biomedical and Health Informatics, vol. 22, no. 1, pp. 108-118, Jan 2018.

[4] P. Li, Y. Wang, Y. Tian, T. Zhou, and J. Li, "An automatic useradapted physical activity classification method using smartphones," IEEE Transactions on Biomedical Engineering, vol. 64, no. 3, pp. 706714, March 2017.

[5] B. Andò, S. Baglio, C. O. Lombardo, and V. Marletta, "A multisensor data-fusion approach for adl and fall classification," IEEE Transactions on Instrumentation and Measurement, vol. 65, no. 9, pp. 1960-1967, Sep. 2016. 


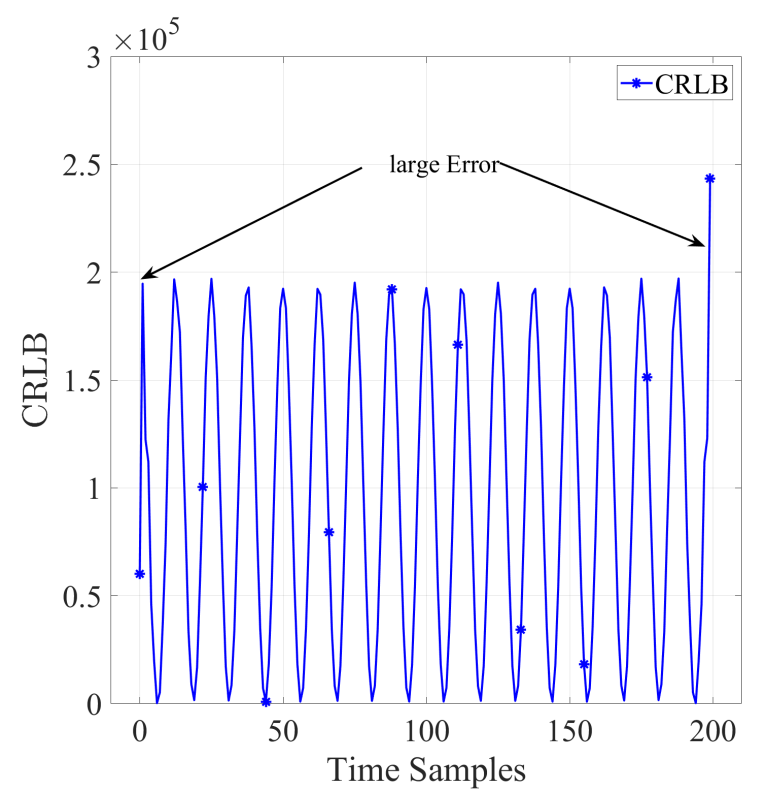

Fig. 11: CRLB at each sample $i$ of the given example.

[6] M. T. Mardini, Y. Iraqi, and N. Agoulmine, "A survey of healthcare monitoring systems for chronically ill patients and elderly," Journal of medical systems, vol. 43, no. 3, p. 50, 2019.

[7] C. Shen, B. Ho, and M. Srivastava, "Milift: Efficient smartwatch-based workout tracking using automatic segmentation," IEEE Transactions on Mobile Computing, vol. 17, no. 7, pp. 1609-1622, July 2018.

[8] T. J. Matarazzo, P. Santi, S. N. Pakzad, K. Carter, C. Ratti, B. Moaveni, C. Osgood, and N. Jacob, "Crowdsensing framework for monitoring bridge vibrations using moving smartphones," Proceedings of the IEEE, vol. 106, no. 4, pp. 577-593, April 2018.

[9] A. A. M. Alzughaibi, "Post-disaster structural health assessment system using personal mobile-phones," Master's thesis, UC Irvine, 2018.

[10] A. A. Alzughaibi, A. M. Ibrahim, A. M. Eltawil, Y. Na, and S. ElTawil, "Post-disaster structural health monitoring system using personal mobile-phones," in 2019 IEEE Topical Conference on Wireless Sensors and Sensor Networks (WiSNet), Jan 2019, pp. 1-4.

[11] Y. Na, S. El-Tawil, A. Ibrahim, and A. Eltawil, "The feasibility of using smart devices for quantifying seismic damage to buildings," in Structures Congress 2017, 2017, pp. 145-154.

[12] Y. Na, S. El-Tawil, A. Ibrahim, and A. Eltawil, "Dynamic behavior of a smart device on a surface subjected to earthquake motion," Earthquake Engineering Structural Dynamics, 2018.

[13] Q. Kong, R. M. Allen, L. Schreier, and Y.-W. Kwon, "Myshake: A smartphone seismic network for earthquake early warning and beyond," Science advances, vol. 2, no. 2, p. e1501055, 2016.

[14] M. Faulkner, M. Olson, R. Chandy, J. Krause, K. M. Chandy, and A. Krause, "The next big one: Detecting earthquakes and other rare events from community-based sensors," in Information Processing in Sensor Networks (IPSN), 2011 10th International Conference on. IEEE, 2011, pp. 13-24.

[15] F. Kalim, J. P. Jeong, and M. U. Ilyas, "Crater: A crowd sensing application to estimate road conditions," IEEE Access, vol. 4, pp. 83178326, 2016.

[16] Y. Mukhtar, V. Nagpal, J. S. Snyder, and C. Walsh, "Systems and methods for detecting mobile device movement within a vehicle using accelerometer data," Jan. 9 2018, uS Patent 9,867,015.

[17] M. J. Manzella and A. Ismaili, "System and method for determining an insurance premium based on analysis of human telematic data and vehicle telematic data," May 29 2018, US Patent 9,984,419.

[18] W. T. Ang, S. Y. Khoo, P. K. Khosla, and C. N. Riviere, "Physical model of a mems accelerometer for low-g motion tracking applications," in Robotics and Automation, 2004. Proceedings. ICRA'04. 2004 IEEE International Conference on, vol. 2. IEEE, 2004, pp. 1345-1351.

[19] G.-X. Liu, L.-F. Shi, J.-H. Xun, S. Chen, L. Zhao, and Y.F. Shi, "An orientation estimation algorithm based on multisource information fusion," Measurement Science and Technology, vol. 29, no. 11, p. 115101, 2018. [Online]. Available: http: //stacks.iop.org/0957-0233/29/i=11/a=115101

[20] A. Ibrahim, A. Eltawil, Y. Na, and S. El-Tawil, "Effect of sensor error on the assessment of seismic building damage," IEEE Transactions on Instrumentation and Measurement, pp. 1-12, 2019.

[21] N. El-Sheimy, H. Hou, and X. Niu, "Analysis and modeling of inertial sensors using allan variance," IEEE Transactions on instrumentation and measurement, vol. 57, no. 1, pp. 140-149, 2008.

[22] C. Maia, L. M. Nogueira, and L. M. Pinho, "Evaluating android os for embedded real-time systems," in 6th international workshop on operating systems platforms for embedded real-time applications, 2010, pp. 63-70.

[23] E. Peguero, M. Labrador, and B. Cook, "Assessing jitter in sensor time series from android mobile devices," in 2016 IEEE International Conference on Smart Computing (SMARTCOMP). IEEE, 2016, pp. $1-8$.

[24] G. Vandersteen and R. Pintelon, "Maximum likelihood estimator for jitter noise models [hf sampling scopes]," IEEE Transactions on Instrumentation and Measurement, vol. 49, no. 6, pp. 1282-1284, 2000.

[25] H. Araghi, M. A. Akhaee, and A. Amini, "Joint compensation of jitter noise and time-shift errors in multichannel sampling system," IEEE Transactions on Instrumentation and Measurement, pp. 1-10, 2018.

[26] S. Ross, A First Course in Probability 8th Edition. Pearson, 2009, ch. 5 , p. 219.

[27] "MPU6500 datasheet," accessed on: February 12th, 2018. [Online]. Available: https://www.invensense.com/wp-content/uploads/ 2015/02/MPU-6500-Datasheet2.pdf

[28] "Piezotronics model 393c datasheet," accessed on: March 11th, 2019. [Online]. Available: http://www.pcb.com/contentstore/docs/PCB_ Corporate/Vibration/Products/Specsheets/393C_P.pdf

[29] G. Gugliandolo, G. Campobello, P. P. Capra, S. Marino, A. Bramanti, G. Di Lorenzo, and N. Donato, "A movement-tremors recorder for patients of neurodegenerative diseases," IEEE Transactions on Instrumentation and Measurement, vol. 68, no. 5, pp. 1451-1457, May 2019.

[30] R. Khusainov, D. Azzi, I. Achumba, and S. Bersch, "Real-time human ambulation, activity, and physiological monitoring: Taxonomy of issues, techniques, applications, challenges and limitations," Sensors, vol. 13, no. 10, pp. 12 852-12902, 2013.

[31] E. K. Antonsson and R. W. Mann, "The frequency content of gait," Journal of biomechanics, vol. 18, no. 1, pp. 39-47, 1985.

[32] N. Fukuwa, R. Nishizaka, S. Yagi, K. Tanaka, and Y. Tamura, "Field measurement of damping and natural frequency of an actual steel-framed building over a wide range of amplitudes," Journal of wind engineering and industrial aerodynamics, vol. 59, no. 2-3, pp. 325-347, 1996.

[33] Y. Xiang, A. Harris, F. Naeim, and F. Zareian, "Identification and validation of natural periods and modal damping ratios for seismic design and building code," in Proceedings of the 2017 Annual Meeting of the Los Angeles Tall Buildings Structural Design Council, 2017, pp. $81-95$.

[34] Y. Ni, X. Lu, and W. Lu, "Operational modal analysis of a highrise multi-function building with dampers by a bayesian approach," Mechanical Systems and Signal Processing, vol. 86, pp. 286-307, 2017.

[35] S. M. Kay, Fundamentals of Statistical Signal Processing: Estimation Theory. Prentice Hall, 1993, vol. 1.

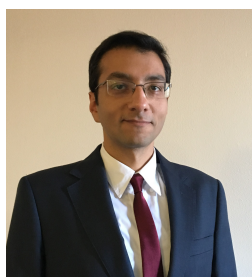

Ahmed Ibrahim received the B.Sc. degree (Hons.) in electrical engineering from Ain Shams University, Cairo, Egypt, in 2008, the M.Sc. degree in microelectronics system design from Nile University, Giza, Egypt, in 2014, and the Ph.D. degree in electrical engineering and computer science from the University of California at Irvine, Irvine, CA, USA, in 2019. From 2008 to 2014, he was a Digital ASIC Design Engineer with Si-Ware Systems, Cairo, where he was involved in designing MEMS sensor interfaces. His research interests include signal processing, machine learning, sensor networks, and sensor fusion. 


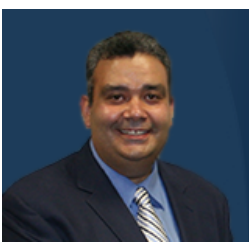

Ahmed Eltawil received the Doctorate degree from the University of California, Los Angeles, in 2003 and the M.Sc. and B.Sc. degrees (with honors) from Cairo University, Giza, Egypt, in 1999 and 1997, respectively. Since 2019 he is a Professor at the Computer, Electrical and Mathematical Science and Engineering Division (CEMSE) at the King Abdullah University of Science and Technology (KAUST), Thuwal, KSA. Since 2005 he was with the Department of Electrical Engineering and Computer Science, at the University of California, Irvine, where he was founder and director of the Wireless Systems and Circuits Laboratory. His research interests are in the general area of low power digital circuit and signal processing architectures with an emphasis on mobile systems. Dr. Eltawil has been on the technical program committees and steering committees for numerous workshops, symposia, and conferences in the areas of low power computing and wireless communication system design. He received several awards, as well as distinguished grants, including the NSF CAREER grant supporting his research in low power systems.

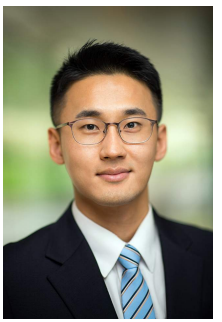

Yunsu Na has received the $\mathrm{PhD}$ degree in Civil and Environmental Engineering from the University of Michigan, Ann Arbor, MI in 2019. He received the M.S. and B.S. degrees from Yonsei University, Seoul, South Korea, in 2013 and 2011 respectively. His research interests include earthquake engineering, computational simulation, reconnaissance system and signal processing.

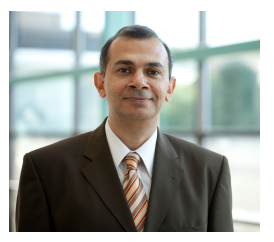

Sherif El-Tawil is the Antoine E. Naaman Collegiate Professor of Civil and Environmental Engineering at the University of Michigan, Ann Arbor, MI. He received his BS and MS degrees from Cairo University in 1989 and 1991 respectively. In 1996, he received the $\mathrm{PhD}$ degree from Cornell University. His research interests include earthquake engineering, community resilience, disaster management, and computational simulation as applied to these topics. 